\title{
Los cuidados al final de la vida: documento de recomendaciones. Una aportación desde la bioética a la calidad de la atención sanitaria en Galicia
}

\author{
Care at the End of Life: Recommendation Statements. \\ A Bioethical Contribution to the Quality of Health Care in Galicia
}

\author{
Juan Antonio Garrido Sanjuán ${ }^{1,2}$ \\ 'Vicepresidente y Coordinador de la línea de trabajo "Cuidados al final de la vida" de la Comisión Galega de Bioética \\ ${ }^{2}$ Servicio de Medicina Interna. Complexo Hospitalario Universitario de Ferrol. SERGAS. Ferrol
}

\section{Resumen}

La Bioética clínica contribuyendo al desarrollo, clarificación y conocimiento del contenido del principio de buena práctica clínica y, al tiempo, incorporando a los actos clínicos los valores de las personas, ha hecho una gran aportación al proceso de toma de decisiones en el delicado periodo del final de la vida. La deliberación bioética, con la incorporación de la prudencia como virtud guía de la reflexión, permite en la mayoría de las situaciones, teniendo en cuenta el marco normativo, deontológico-profesional y legislativo, tomar decisiones respetuosas con la autonomía de las personas.

Hay personas que mueren en condiciones inaceptables, desde el punto de vista de la atención al dolor y al sufrimiento y de un insuficiente respeto a su autonomía, en una sociedad que, como la nuestra, ha alcanzado importantes niveles de desarrollo sanitario, cultural, legal, político y económico. La Comisión Galega de Bioética, a partir de la preocupación por esta realidad y de los pasos dados ya en nuestra Comunidad Autónoma para tratar de mejorarla, ha elaborado un documento de recomendaciones. Su objetivo es servir como marco de referencia que oriente la reflexión de los profesionales y usuarios en los conflictos éticos que surgen en los cuidados al final de la vida, y contribuir a mejorar el desarrollo de los derechos fundamentales en este ámbito. El conocimiento y puesta en práctica de estas recomendaciones debería conducir a conseguir una gestión más autónoma, con mayor equidad y con menor sufrimiento del proceso del final de la vida. En definitiva, alcanzar una atención de calidad al final de la vida para toda la población.

Se plantean las recomendaciones como llamadas de atención 0 descripción de los pasos que deben darse o reforzarse para hacer posible el cumplimiento de las acciones 0 actuaciones de buena práctica clínica, definidas en el marco conceptual, que no sólo son posibles respetando la normativa actual (también recogida en el documento) sino obligadas para el desarrollo y respeto de los derechos de los ciudadanos en este campo.

\section{Introducción}

Este artículo tiene como finalidad servir de presentación y ayudar a la difusión de los contenidos del Documento de Recomendaciones sobre los cuidados al final de la vida elaborado por la actual Comisión Galega de Bioética. Incide en algunos de los ejes que guían las recomendaciones y en el marco conceptual que las engloba, tratando de sugerir como puede ser utilizado por los distintos destinatarios del mismo.

La publicación se presentó en una Jornada cuyo título, "Bioética un instrumento de calidad para la atención sanitaria en el Sistema Público de Salud de Galicia", tiene una clara vinculación con el desarrollo y la finalidad buscada por el documento, motivo por lo que dedicaremos un espacio inicial a justificar esta afirmación.

\section{En síntesis la finalidad y contenidos del artículo son:}

- Justificar el trabajo que dio lugar al documento en un horizonte más amplio que coloca la Bioética como uno de los ejes de la mejora de la calidad en la asistencia sanitaria.

- Comunicar los antecedentes y el por qué de la elaboración de este Documento.

- Resaltar los contenidos o conceptos "novedosos" que tratan de captar más la atención de los que se acerquen al documento, como medio de estimular una nueva reflexión y vías o cursos de acción.

- Llamar la atención sobre el importante papel ejercido por la Medicina Interna y los internistas en la atención sanitaria al final de la vida y su posición idónea, junto a los profesionales de atención primaria, para hacer posibles o consolidar las recomendaciones que propone el documento.

- Sugerir como puede ser utilizado para que pueda ayudar a los destinatarios en los distintos ámbitos: pacientes y allegados, profesionales del sistema sanitario, Comités de ética asistencial, equipos directivos, Administración... 


\section{1. ¿Cómo nos puede ayudar}

\section{la bioética a mejorar la asistencia sanitaria a nuestros pacientes?}

En las últimas décadas se han producido cambios en la práctica clínica que explican la fuente de los principales conflictos éticos. Por un lado, se ha producido un cambio en las relaciones sanitarias, pasando de una relación paternalista en la que el médico decidía y el paciente obedecía, a una relación menos asimétrica entre dos personas responsables y autónomas. En esta relación se hace necesario incorporar, a la hora de tomar decisiones, la diversidad de valores de las personas en una sociedad, como la nuestra, plural. Por otro lado los grandes avances científico-técnicos nos llevan a cuestionarnos si se debe hacer todo lo técnicamente posible, todo lo que se puede conseguir tecnológicamente en cada situación. El tercer elemento implicado es el acceso igualitario a la Sanidad y la obligación de hacer una distribución equitativa de unos recursos que son limitados. En este escenario, para prestar una asistencia de calidad nuestros pacientes no basta con una buena formación científico-técnica. Esta es una condición necesaria pero no suficiente. Es preciso introducir en nuestros razonamientos el respeto a la autonomía de las personas, la cual no puede quedar invalidada por el hecho de vivir una situación de vulnerabilidad como es la enfermedad.

La Bioética es una ética aplicada que introduce en el mundo sanitario una relación clínica ligada a una sociedad democrática que, por una parte, exige el respeto a la autonomía del ciudadano, y al mismo tiempo sitúa el acceso a la asistencia sanitaria como una cuestión de justicia distributiva, que la sociedad debe procurar garantizar a todos los individuos. La Bioética clínica ayuda a identificar y a potenciar dentro de los distintos modelos de relación clínica aquellos que permiten el respeto de los pacientes y sus derechos. El conocimiento y aplicación de la Bioética ayuda al desarrollo de un proceso de consentimiento informado de excelencia.

La formación en Bioética resulta fundamental, por tanto, como ayuda al clínico para la resolución de conflictos éticos. Pero además, de forma trasversal, ayuda a mejorar otros aspectos de la asistencia clínica diaria. Por una parte, al aplicar la metodología bioética centrada en la deliberación prudente, siempre comenzamos con el análisis de los hechos, de los datos clínicos de un paciente; al hacer esto, a veces, nos encontramos con que no estamos ante problemas éticos sino ante errores técnicos y su identificación nos puede ayudar a corregirlos. En este sentido el análisis ético actúa como un estimulo para la formación continuada y, por tanto, contribuye a mejorar la formación científico-técnica. Por otra parte la Bioética estimula la formación del profesional en habilidades de comunicación. Estas son herramientas fundamentales para la aplicación real del consentimiento informado, para resolver las situaciones de conflicto y otras cuestiones relacionadas directamente con la Bioética, como puede ser la valoración de la capacidad.

La Bioética clínica contribuyendo al desarrollo, clarificación y conocimiento del contenido del principio de buena práctica clínica (equivalente al clásico principio de no maleficencia), y al tiempo incorporando a los actos clínicos los valores de las personas, ha hecho una gran aportación al proceso de toma de decisiones en el delicado periodo del final de la vida.

La deliberación bioética, con la incorporación de la prudencia como virtud guía de la reflexión, permite, en la mayoría de las situaciones, teniendo en cuenta el marco normativo, deontológico-profesional y legislativo, tomar decisiones respetuosas con la autonomía de las personas incorporando en las mismas las buenas prácticas profesionales relacionadas con la fragilidad y el potencial sufrimiento en esta fase de proximidad de la muerte.

\section{El por qué de la elección de esta línea de trabajo. Antecedentes}

A pesar de los progresos producidos en las últimas décadas no es una realidad excepcional en el ámbito sanitario el insuficiente respeto a la autonomía de las personas en el proceso de morir. Tampoco es infrecuente la carencia de atención y cuidados sanitarios adecuados a las personas en esta etapa vital para, en el marco de la atención paliativa, posibilitar su evolución con los menores niveles posibles de dolor y sufrimiento.

Como dice el documento en su introducción, "Se ha llegado al punto en que dentro de la sociedad hay quienes tienen la percepción errónea de que la muerte casi siempre puede y debe ser pospuesta. Y no solo la sociedad, pues una parte de los profesionales y los diferentes sistemas de salud en los que se encuadran, prestan poca atención a las necesidades de las personas sin posibilidades de curación y dedican la mayor parte de los recursos al diagnóstico y al tratamiento curativo de las enfermedades, olvidando que una de sus misiones es aliviar el sufrimiento humano.

Este enfoque de la medicina es uno de los responsables de que muchos profesionales sanitarios se encuentren incómodos e inseguros ante el final de la enfermedad sin saber bien como actuar. Existen dificultades para reconocer que esta etapa ha llegado y, en ocasiones, falta el coraje necesario para actuar correctamente. Se produce un inadecuado control de los síntomas; hay dudas y miedo para retirar o no aplicar determinados tratamientos, y se dan asimismo dificultades de comunicación e incomprensión ante la diversidad cultural y de creencias.

Como consecuencia de ello, se constatan actitudes de abandono del paciente 0 , por el contrario, actuaciones encami- 
nadas a prolongar la vida, más allá de lo que puede considerarse compatible con una existencia en condiciones de dignidad personal."

De forma esquemática podemos decir que:

A) este enfoque es responsable de que muchos profesionales:

- Se encuentren inseguros ante el final de la vida

- Tengan dificultades para reconocer que esta etapa ha llegado

- En ocasiones les falte el "coraje" para actuar correctamente

- Tengan dudas y miedo para retirar o no aplicar tratamientos 0 incluir esta posibilidad entre las posibles decisiones

B) en nuestra sociedad todavía hay personas que mueren:

- Solas

- Sin control sintomático adecuado

- Recibiendo acciones o procedimientos:

- No consultados o no deseados

- No indicados: aquello con baja probabilidad de éxito o no claras evidencias de beneficio

- E incluso ii Contraindicados !! (todo lo que prolonga la agonía está contraindicado)

En relación con el hecho de que todavía hay personas que mueren siendo sometidas a acciones o procedimientos no consultados o no deseados, es una realidad en nuestra sociedad el escaso conocimiento de la posibilidad de realizar un proceso de planificación anticipada de las decisiones y un documento de instrucciones previas como final de este proceso. El dato del escaso número de estos documentos realizados en nuestra comunidad autónoma, y menos aún registrados, apoya esta afirmación. Estos documentos realizados son escasamente consultados por los profesionales, según reconocen profesionales de urgencias y medicina intensiva. No sabemos además si los pocos existentes, si son consultados, llegan a ser suficientemente respetados y existen problemas también en el conocimiento y/o respeto de los otros criterios de aplicación del juicio sustitutivo (respetar las decisiones del representante, criterio del mejor interés, etc...), cuando no existen documentos de instrucciones previas.

En relación a la afirmación de que en nuestra sociedad todavía hay personas que mueren recibiendo acciones o procedimientos no indicados o incluso contraindicados, podemos encontrar distintos factores implicados en estas conductas inadecuadas de los profesionales:

- La falta de formación de los profesionales: confusiones conceptuales (sedación versus eutanasia, limitación de esfuerzo terapéutico versus eutanasia....), dificultades técnicas en la indicación y/o realización de sedación, etc.

- La falta de conocimientos 0 errores en la interpretación del marco jurídico: tiene como consecuencia dificultades en el respecto a la autonomía del paciente y de su derecho a decidir al final de la vida.
- La incomprensión por parte de otros implicados en el proceso como son otros profesionales, familiares, responsables de las instituciones... etc.

- La falta de apoyo y compromiso institucional.

- La falta de cultura social sobre la vulnerabilidad y fragilidad del ser humano y la finitud de la existencia humana.

En resumen, en nuestra sociedad todavía hay personas que mueren con un inadecuado cuidado a la especial fragilidad y vulnerabilidad de la persona moribunda, y siguen existiendo dificultades en el mantenimiento de la equidad en el acceso a la atención de calidad en el final de la vida. Hay, asimismo, una falta de autonomía en la gestión de esta fase de la vida y esto es debido, en parte, a actuaciones profesionales mejorables y, por otro lado, a deficiencias en la organización y en el apoyo de la Administración a los profesionales.

La Comisión Galega de Bioética, en su preocupación por estos problemas éticos que se suscitan al final de la vida, y conocedora de la situación descrita, en su afán por intentar cambiarla, ha impulsado un proceso de reflexión cuyo resultado es el Documento de Recomendaciones al que este texto hace referencia.

\section{Objetivo del documento}

Su objetivo es servir de marco de referencia y orientación a profesionales y usuarios sobre los conflictos éticos que surgen en esta fase de la vida y contribuir a mejorar el desarrollo de los derechos fundamentales en este ámbito.

\section{Método}

El documento ha sido fruto de un proceso reflexivo y deliberativo partiendo de la síntesis de una revisión bibliográfica no estructurada y de las aportaciones de los miembros de la Comisión.

\section{5. ¿Qué contenidos vais a encontrar en el documento?}

(http://www.sergas.es/Docs/Bioetica/Cuidados\%20CAST_240912_link.pdf)

- Presentación.

- Introducción.

- Objetivo.

- Recomendaciones:

- Relacionadas con la calidad de la atención que permite una gestión más autónoma de proceso del final de la vida.

- Relacionadas con la equidad en el acceso a la atención de calidad al final de la vida.

- Marco conceptual y terminológico:

- Aspectos éticos.

- Autonomía del paciente.

- Situaciones clínicas.

- Anexos:

- I-Documentación

- II - Normativa comentada 


\section{Principales aportaciones del documento}

Este artículo no pretende sustituir a la lectura del documento sino animar a la misma y su objetivo no es entrar en el enunciado pormenorizado de las recomendaciones. Sin embargo, sí quisiera resaltar las que se pueden considerar como principales aportaciones del documento desde nuestro punto de vista.

A) El documento recoge los pasos dados previamente con el objetivo de mejorar la calidad de la atención al final de la vida en nuestra Comunidad Autónoma:

- El desarrollo de los Comités de Ética Asistencial en los distintos centros 0 áreas sanitarias, que han recogido y manifestado su preocupación por las deficiencias en este campo y la inquietud por colaborar en su mejora. Prueba de ello son los múltiples documentos vinculados con la inquietud por distintos aspectos de los cuidados al final de la vida desarrollados por los comités de ética asistencial de nuestra Comunidad Autónoma.

- El Plan Galego de Coidados Paliativos que fue definido en 2006 como aquel que desarrolla la atención a pacientes diagnosticados de enfermedades, oncológicas y/o no oncológicas, progresivas o en estado avanzado, en los que, dado que pronóstico de vida limitado, la prioridad es proporcionar los cuidados necesarios para atender las necesidades físicas, psicosociales y espirituales del paciente y de su familia.

- El desarrollo legislativo de los documentos de instrucciones previas sobre cuidados y tratamiento de la salud y el Registro gallego de instrucciones previas, creado en diciembre de 2007, después de haberse constituido ese mismo año el registro estatal. En el año 2008, se desarrolló una campaña informativa y se puso a disposición de los profesionales y pacientes una guía de instrucciones previas sobres cuidados y tratamiento de la salud. Dicha guía ya recomendaba fomentar la planificación anticipada de las instrucciones previas y no solo el desarrollo de los documentos.

- La Estratexia SERGAS 2014: Plan estratégico que incluye entre sus objetivos potenciar la atención y los cuidados al paciente en el final de su vida

B) Marco conceptual y terminológico. Las palabras y el lenguaje no son meros cauces de expresión para la persona que habla o escribe, sino potentes instrumentos para acceder a la realidad de lo que son las cosas. El correcto uso de los términos facilita la compresión de las acciones a que éstos se refieren y pretende ser una herramienta para los profesionales en la práctica asistencial que propicie procesos de deliberación moral cuyo resultado tenga sentido para quienes participen en él y para los que se hallen afectados por el mismo.

El marco conceptual y terminológico se ha concebido como algo más allá que un simple glosario de términos, puesto que incluye la valoración ética y normativa vinculada a esos conceptos, conocimiento que es necesario para el impulso de las recomendaciones.
Dentro de este pormenorizado marco conceptual, me detendré en algunos términos que se proponen y/o refuerzan para llamar la atención sobre las prácticas que los mismos encierran.

C) Documentos recomendados: Se incluyen aquí algunos documentos de recomendaciones, guías o protocolos realizados por otras comisiones, comités o grupos de trabajo, que la Comisión Galega de Bioética considera pueden ayudar a los profesionales a llevar a la práctica las recomendaciones propuestas y, por tanto, ayudar a conseguir una atención de calidad al final de la vida.

D) Normativa comentada: En la actualidad se ha alcanzado un consenso ético y jurídico básico entorno a algunos de los contenidos y los derechos del ideal de buena muerte. No obstante, persiste cierta dificultad para llevar a la práctica clínica las decisiones tendentes a asegurar de manera efectiva la plena dignidad en el proceso de morir. Se invoca un supuesto "vacío legal" para justificar dicha dificultad, pero no es más que una percepción errónea de la realidad. El ordenamiento jurídico proporciona un marco orientativo y de amparo suficiente para resolver las situaciones complejas que se producen en el proceso de morir. No obstante, debe admitirse la conveniencia de hacer un esfuerzo de armonización y concreción legislativa que sistematice la materia, establezca las garantías de su aplicación y, en la mayor medida posible, facilite su manejo y comprensión por parte de todos los implicados.

No es buena práctica clínica alargar la vida a cualquier precio y, puesto que el hecho de la muerte es inevitable, el mantenimiento de la vida no debe considerarse más importante que lograr una muerte en paz, evitando el sufrimiento innecesario.

\section{Algunos conceptos a destacar dentro del marco conceptual}

A) Adecuación de intervenciones clínicas: la utilización de este término podría contrarrestar en algún caso el rechazo que produce a veces el término limitación (entendiendo sin embargo que la limitación es una actuación perfectamente válida profesional y éticamente y a veces de cumplimiento obligado). Pretente resaltar la derivación de esfuerzos, más que la limitación, hacia otra parcela (los cuidados). Implica ofertar solo aquellos procedimientos diagnósticos o terapéuticos proporcionados al pronóstico y momento evolutivo de la enfermedad, y llevar a cabo únicamente aquellos consentidos por el paciente 0 su representante.

B) Limitación del esfuerzo terapéutico: se trata de la decisión deliberada o meditada del médico responsable del paciente, que consiste en no instaurar o retirar medi- 
das terapéuticas que no le aportarán un beneficio claro cuando se percibe una desproporción entre los fines y los medios terapéuticos (entre ambas formas de hacerlo, retirar o no instaurar, hay equivalencia moral). Permite a la enfermedad continuar su curso evolutivo que inexorablemente conduce al fallecimiento de la persona. La limitación del esfuerzo terapéutico forma parte de la "buena práctica clínica". No es una decisión opcional sino una obligación moral y normativa de los profesionales.

C) Tratamiento o medida de soporte vital: cualquier intervención médica, técnica, procedimiento o medicación que se administra a un paciente con el fin de retrasar el momento de la muerte, esté dicho tratamiento o no dirigido hacia la enfermedad de base 0 al proceso biológico causal. Es decir, cualquier tratamiento con capacidad para prolongar la vida. Incluye ventilación mecánica, diálisis, transfusiones, etc. El documento destaca que en la actualidad hay un amplio consenso para considerar la nutrición e hidratación artificial como medidas de soporte vital a la hora de la deliberación sobre decisiones de limitación de esfuerzo terapéutico.

D) Sedación paliativa: el documento subraya que se trata de una práctica permitida por nuestro ordenamiento jurídico, y su realización, cuando se cumplen las condiciones descritas en el marco conceptual, entra dentro de las obligaciones de buena práctica clínica. Siempre que se realice una sedación se debe dejar constancia en la historia clínica del motivo, el proceso de toma de decisiones, el consentimiento informado y la monitorización de los resultados, siendo este registro la mejor garantía ética.

E Actuaciones clínicas limitadas en el tiempo: El documento plantea este concepto como ejemplo de desarrollo de una actitud deliberativa y aplicación de un curso de acción intermedio en las decisiones al final de la vida. Se trata de la descripción de una forma de actuar comunicada recientemente (JAMA. 2011; 306:1483-1484). Consiste en un acuerdo entre los profesionales sanitarios y el paciente (o su representante en caso de incapacidad) sobre la utilización de ciertos procedimientos médicos durante un periodo definido de tiempo, evaluando la respuesta según criterios consensuados que determinarán la continuidad o suspensión del procedimiento transcurrido ese tiempo. Aunque los autores de la propuesta hablan de tratamientos 0 intentos terapéuticos ("Time-limited trials near the end of life"), preferimos utilizar el término actuaciones clínicas porque creemos que el mismo abordaje puede ser adecuado para determinadas acciones diagnósticas y no sólo terapéuticas.
La elaboración de estrategias anticipadas y consensuadas de cuidados y tratamientos, como propone el procedimiento de actuaciones clínicas limitadas en el tiempo, puede permitir a las familias aprender y observar como los médicos están tratando de balancear los beneficios y cargas del tratamiento con honestidad y compasión con el paciente y la familia. Asimismo puede ayudar a los miembros del equipo a llegar a un consenso y hablar con una sola voz, y a reducir los conflictos potenciales entre los equipos sanitarios y el paciente 0 la familia mediante el establecimiento de las expectativas mutuas y un diálogo regular y estructurado sobre la evolución. Facilitará establecer un proceso intermedio entre los pacientes 0 familias que quieren "todo" y los profesionales que pueden limitar unilateralmente el tratamiento.

F) Decisiones compartidas: Se trata de un estilo de relación mediante el cual el profesional aplica conocimientos científicos y habilidades de comunicación, teniendo en cuenta los valores y preferencias del paciente para ayudarle y apoyarle en su decisión. Incluye el esfuerzo por conocer las circunstancias específicas del paciente, sus necesidades y valores, y supone un proceso de diálogo y compromiso compartido. Se distingue del modelo "paternalista" en que, en este último, es el médico quien, basándose en sus conocimientos científicos, decide lo que considera mejor para el paciente, pero sin contar con él. También se diferencia del modelo "informativo" en el cual el paciente, tras ser informado, decide solo, sin ayuda del profesional.

G) Continuidad asistencial/fragmentación de cuidados: Se entiende por continuidad asistencial la coherencia y unión de las experiencias en la atención percibidas por el paciente a lo largo del tiempo. Conforme a las normas de buena práctica clínica, el paciente y sus cuidadores serán atendidos en los dispositivos adecuados al nivel de complejidad de su situación clínica. Fomentar el trabajo en equipo, y que sus miembros compartan las incertidumbres y decisiones, favorece la continuidad asistencial.

\section{Algunos comentarios sobre las recomendaciones enunciadas en el documento:}

Quisieramos en este punto dedicar un momento a destacar las líneas seguidas por los contenidos de algunas de las recomendaciones, 0 incluso detenernos en alguna de las recomendaciones. Estos comentarios no pretenden destacar como más importantes éstas, respecto a otras recomendaciones que no van a ser comentadas, sino simplemente hacer una llamada de atención por ser contenidos más difíciles de encontrar 0 ausentes en otros documentos similares. 
- La relación de cercanía de los profesionales sanitarios que desempeñan sus funciones en la atención primaria de salud con sus pacientes, los sitúa en una posición idónea para favorecer la reflexión sobre cuestiones vinculadas con el final de la vida.

- En relación con los documentos de instrucciones previas se insiste en la importancia de que los profesionales sanitarios informen a los pacientes sobre la utilidad de su realización y les ayuden a definir situaciones futuras en la evolución de la enfermedad, fomentando el uso de los documentos en un contexto de planificación anticipada de los cuidados. Asimismo se insta a la Administración a mejorar la accesibilidad para realizar y registrar los documentos de instrucciones previas.

- Se afirma que, en un contexto de enfermedad avanzada con un pronóstico de vida limitado, la indicación de no reanimación cardiopulmonar debe considerarse el estándar de buena práctica clínica.

- Se hace mención a la necesidad de la atención espiritual que además debe estar en consonancia con las convicciones y creencias de la persona.

- Se destaca la importancia de la formación de los profesionales, no solo como medio de garantizar la calidad de la atención en esta fase de sla vida, sino también como medio de garantizar la equidad y el respeto de los derechos sanitarios de las personas atendidas en distintos ámbitos y dispositivos.

- Se resalta la importancia de promover actividades de mejora continua de la calidad en los centros sociosanitarios, residencias de personas mayores y dispositivos de atención urgente, con la finalidad de mejorar los cuidados y disminuir las actuaciones desproporcionadas y los traslados inadecuados a centros sanitarios de atención de procesos agudos.

- Se llama la atención sobre el esfuerzo que deben realizar los profesionales y la organización para lograr una efectiva continuidad asistencial que evite la fragmentación de los cuidados.

- Se recomienda desarrollar en el campo de la gestión sanitaria indicadores que permitan medir la actividad asistencial y monitorizar la calidad de la atención en el proceso del final de la vida.

- El ordenamiento jurídico proporciona un marco orientativo y de amparo suficiente para resolver situaciones complejas en la práctica clínica. Aún así, se recomienda elaborar guías que mejoren no sólo la calidad asistencial sino la seguridad jurídica.

\section{Los internistas y los cuidados al final de la vida}

Como dice la guía de formación de la especialidad de Medicina Interna, "Ios internistas se forman bajo el principio de que ninguna enfermedad o problema clínico del adulto es ajeno a su incumbencia y responsabilidad. Esta actitud les ha llevado a participar activamente en la mayoría de los retos asistenciales a los que ha tenido que hacer frente nuestro sistema sanitario". Este hecho puede verificarse cuando revisamos su presencia en los distintos campos asistenciales que, con una perspectiva más o menos cercana al final de la vida, se han ido desarrollando: atención al paciente anciano, enfermedades crónicas y degenerativas, pacientes pluripatológicos, oncología, planes y unidades de cuidados paliativos.

El concepto de internista como "especialista en la persona enferma" quiere resaltar la importancia de la atención a la persona y no a la enfermedad, cualidad ésta que, siendo importante que aparezca en toda la relación clínica, se hace esencial que esté presente en el médico cuando la enfermedad está conduciendo a la persona al final de su vida.

También en la guía de formación de la especialidad se recoge que "el papel del internista como garante de la atención integral en el entorno hospitalario es decisivo para favorecer una atención sanitaria centrada en las necesidades globales de la persona. Con ello se evita el riesgo de atomizar la medicina no haciendo de la tecnología el eje exclusivo de la atención hospitalaria". Como queda recogido en el Documento de Recomendaciones que estamos presentando, es necesaria, especialmente en el ámbito hospitalario excesivamente mediatizado por la disponibilidad de medios diagnósticos y terapéuticos de más alto desarrollo tecnológico, la presencia de profesionales que, especialmente en el ámbito del final de la vida, introduzcan la reflexión sobre la "adecuación de las intervenciones clínicas" según se define previamente en esta publicación. Su formación y visión integral del paciente deben permitirle introducir en la deliberación con otros profesionales del hospital las decisiones de no instauración o retirada de medidas terapéuticas, en contextos con riesgo de conducir a conductas diagnósticas o terapéuticas desproporcionadas.

Por su formación en la importancia de la historia clínica, mediada por una buena comunicación con el paciente, de la información en el desarrollo de la relación clínica, y por su vínculo prolongado en el tiempo con los pacientes crónicos, el internista también debe ocupar un lugar prioritario en el desarrollo de los procesos de planificación anticipada de las decisiones que el Documento recomienda potenciar como medio de mejorar la calidad de la atención al final de la vida.

\section{Sugerencias sobre cómo utilizar y difundir el Documento de Recomendaciones}

A) A los pacientes y allegados el documento les puede permitir conocer mejor aquello que los profesionales y la Administración debemos, y tendríamos que poder, proporcionarles. Debe estimular la búsqueda, y si es necesario la reclamación, de aquello que se recoge en el 
documento y a lo que la Administración se compromete.

B) A los profesionales debería suponerles un sustento profesional, ético y legal para la toma de decisiones difíciles en este campo de actuación.

C) A los comités de ética asistencial les debería permitir trabajar, desarrollar y demandar el desarrollo de los medios necesarios para que se cumplan las recomendaciones, en los distintos centros en que están constituidos, y además ser un elemento de ayuda en la resolución de los conflictos que lleguen hasta ellos.

D) Para los equipos directivos asistenciales debería ser una herramienta para apoyar a los profesionales, para dar respuesta a la demanda de los pacientes y para demandar medios a la Administración.

E) Todos los implicados podemos utilizarlo para realizar una lectura reflexiva, con evaluación constructiva de lo existente frente a lo recomendado, frente a lo que debería ser. Además los documentos recomendados existentes previamente, cuyo acceso es facilitado por esta publicación, deberían ser una apoyo para la toma concreta de decisiones.

\section{Para finalizar}

El desarrollo de las recomendaciones descritas en este documento debería conseguir que:

- Ninguna persona en estado terminal sea tratada contra su voluntad.

- El paciente, si lo desea, tenga información compresible y veraz sobre su diagnóstico y pronóstico.

- Existan las condiciones en los centros sanitarios que faciliten la presencia de personas con las que mantenga vínculos familiares o de hecho en los procesos que requieran la hospitalización de las personas en estado terminal.

- Estén desarrollados y protegidos los procesos de toma de decisiones por representación en caso de pacientes incapaces.

- Se garantice la continuidad asistencial especialmente en la atención al final de la vida.

- Toda la población gallega tenga acceso y reciba cuidados paliativos óptimos según los estándares europeos.

- Que la Administración establezca los mecanismos necesarios para el reconocimiento del esfuerzo dedicado por los profesionales a la mejora de los complejos procesos de comunicación y decisiones al final de la vida y que procure, para proporcionarles seguridad profesional y jurídica, que estén elaboradas y sean accesibles en todos los centros guías de práctica clínica relacionadas con:

a) cuidados paliativos y sedación,

b) limitación del esfuerzo terapéutico,

c) aplicación de decisiones de sustitución y abordaje de situaciones conflictivas derivadas de estas decisiones, incluyendo la implicación de los Comités de Ética Asistencial en las distintas fases de estos procesos.

Terminamos con unas palabras que incluye el presidente de la Comisión en la presentación del documento, como reflejo del compromiso que adquiere la Administración al aceptar y difundir el Documento de Recomendaciones elaborado por la Comisión Galega de Bioética:

"Para el Servicio Gallego de Salud, dotar a los profesionales del apoyo, la información y las herramientas necesarias para asumir su papel con seguridad y reducir la conflictividad en una situación dolorosa y compleja como el final de la vida, constituye una obligación ineludible con ellos, y un compromiso con la ciudadanía".

Nota del autor: para la elaboración de este artículo se ha utilizado en gran parte el texto uilizado por el autor en la presentación del Documento de Recomendaciones sobre los cuidados al final de la vida, en el seno de la Jornada "Bioética un instrumento de calidad para la atención sanitaria en el Sistema Público de Salud de Galicia" celebrada el 25 de octubre de 2012 en el salón de actos de la Consellería de Sanidade en Santiago de Compostela.

\section{Bibliografía}

Además de la bibliografía descrita en el documento presentado (http:// www.sergas.es/Docs/Bioetica/Cuidados\%20CAST_240912_link.pdf), se ha utilizado para este artículo la siguiente bibliografía:

1. Ameneiros E, Carballada C, Garrido JA. ¿Cómo nos puede ayudar la bioética a mejorar la asistencia sanitaria a nuestros pacientes? Galicia Clín 2009;70:27-8.

2. Couceiro A. Aprendizaje práctico de la bioética en el pregrado: objetivos, herramientas docentes y metodología. Educ Med 2012;15:79-87.

3. Garrido JA. El internista y las competencias en el área de la bioética. En: Sociedad Galega de Medicina Interna, edit. La Medicina Interna como modelo de práctica clínica. A Coruña. 2008:231-246.

4. Gracia D. La deliberación moral: el método de la ética clínica. Med Clin (Barc) 2001; 117: 18-23.

5. Jonsen A. La ética en la práctica de la medicina interna. En: Couceiro A. edit. Bioética para clínicos. Madrid: Triacastela; 1999:223-232

6. Orden $\mathrm{SCO} / 227 / 2007$, de 24 de enero, por la que se aprueba y publica el programa formativo de la especialidad de Medicina Interna. BOE de 7 de febrero de 2007. 